\title{
Changes in long term survival after diagnosis with common hematologic malignancies in the early 21st century
}

\author{
Dianne Pulte' ${ }^{1}$ Lina Jansen ${ }^{1}$ and Hermann Brenner ${ }^{1,2,3}$
}

\begin{abstract}
Five-year survival has increased for many hematologic malignancies in the 21st century. However, whether this has translated into greater long-term survival is unknown. Here, we examine 10- and 20-year survival for patients with multiple myeloma (MM), acute lymphoblastic leukemia (ALL), acute myeloblastic leukemia (AML), chronic lymphoid leukemia (CLL), chronic myeloid leukemia (CML), non-Hodgkin lymphoma (NHL), and Hodgkin lymphoma (HL). Data were extracted from the Surveillance, Epidemiology, and End Results-9 database. Patients age 15+ with the above malignancies were included. The newly developed boomerang method was used to examine 10- and 20-year relative survival (RS) for patients in 2002-2006 and 2012-16. Ten and 20-year RS increased for each malignancy examined, with increases ranging from $+4.4 \%$ units for 20 -year RS for AML to $+23.1 \%$ units for 10-year RS for CML. Ten year RS was $>50 \%$ in 2012-16 for patients with CLL, CML, $\mathrm{HL}, \mathrm{NHL}$, and $\mathrm{DLBCL}$, at $77.1 \%, 62.1 \%, 63.9 \%, 64.5 \%$, and 63.0\%,

respectively. Survival dropped between 10 and 20 years after diagnosis for most malignancies. Long-term survival is increasing for common hematologic malignancies, but late mortality is an ongoing issue. Further study of long-term outcomes in curable malignancies to determine the reason for these later decreases in survival is indicated.
\end{abstract}

\section{Background}

Recent progress in the treatment of many hematologic malignancies has resulted in improved population level 10-year survival ${ }^{1}$, particularly chronic myeloid leukemia $(\mathrm{CML})^{2,3}$, chronic lymphocytic leukemia $(\mathrm{CLL})^{4}$, multiple myeloma $(\mathrm{MM})^{5,6}$, and some subtypes of non-Hodgkin lymphoma $(\mathrm{NHL})^{7}$. However, how these changes have effected longer term survival on the population level is less well documented.

Documentation of changes in longer term survival is hampered by the fact that improved survival for many malignancies has occurred only due to changes in therapy that may have occurred only in the past decade and

Correspondence: Dianne Pulte (pultedi@gmail.com)

${ }^{1}$ Division of Clinical Epidemiology and Aging Research, German Cancer Research Center (DKFZ), Heidelberg, Germany

${ }^{2}$ Division of Preventive Oncology, German Cancer Research Center (DKFZ) and National Center for Tumor Diseases (NCT), Heidelberg, Germany

Full list of author information is available at the end of the article analysis of longer term survival will necessarily include data from patients treated prior to the availability of these therapies. For example, tyrosine kinase inhibitors (TKI) drastically changed the natural history of CML and the first TKI was approved in the United States (US) only in $2001^{8}$. Thus, any estimate of, for example, 20 year survival will necessarily be an underestimate, even using period analysis, a method that has been shown to provide more up-to-date survival estimates than traditional cohort-based survival analysis methods ${ }^{9}$. Recently, a new method of survival analysis, the boomerang method ${ }^{10}$, was proposed to further enhance up-to-dateness of long-term survival estimates. This method was shown empirically to provide long-term survival estimates closer to those later observed when survival is changing rapidly, i.e. when survival improves drastically due to new therapeutic options.

Here, we examine 10- and 20-year survival for patients with common hematologic malignancies in the United States (US) using the boomerang method. 


\section{Methods}

Data were extracted from the Surveillance, Epidemiology, and End Results (SEER)9 database. The SEER9 database includes data from nine regional cancer registries throughout the US. Registries are chosen for their high quality and epidemiologically significant populations ${ }^{11}$. The SEER9 registries provide data on cancer survival starting in 1973 and thus are ideal sources of data for examination of long term population level survival. The population within the SEER registry is similar to the general US population in most respects, although there is deliberate oversampling of some minority ethnic groups and a higher proportion of foreign-born persons than in the general US population ${ }^{11}$. Adults with a diagnosis of myeloma, acute lymphoblastic leukemia (ALL), acute myeloblastic leukemia (AML), CLL, CML, Hodgkin lymphoma (HL) and NHL selected by ICD-10 codes C90.0 (MM), C91.0 (ALL), C92.0, C92.3, C92.4, C92.5, C92.6, C92.8, and C92.9 (AML), C91.1 (CLL), C92.1 (CML), C81 (HL), and C82-86 (NHL), were included in the analysis. Because NHL is a very heterogeneous condition with multiple subtypes with varying survival expectations, an analysis on the most common type of NHL, diffuse large B-cell lymphoma (DLBCL) was performed as well, with cases selected using the ICD-10 code C83.3. Cases diagnosed by death certificate only (DCO) were excluded.

Ten and twenty-year survival for patients diagnosed with the above malignancies in 2002-2006 and 2012-16 were estimated using the boomerang method. The boomerang method of estimating survival has been described in detail elsewhere ${ }^{10}$. Briefly, with the boomerang approach, the survival experience in the initial 5 years for a given time of diagnosis is obtained by a complete analysis of survival for patients diagnosed in the most recent calendar years, whereas survival for later years (i.e., 6-10 or 6-20 years) after diagnosis is contributed by the survival experience diagnosed in earlier years in a "period-like" approach, minimizing the contribution of the survival experience of patients diagnosed in earlier calendar periods (see Supplemental Fig. 1). This method has been demonstrated to produce survival estimates closer to those later observed for cancers where survival is changing rapidly, i.e. those where new treatment options have changed the survival expectations.

According to common practice in population-based cancer survival studies, relative, rather than absolute, survival was assessed. Relative survival (RS) estimates are obtained by taking the ratio of absolute survival rates compared to the expected survival rates for a similar group of people in the general population. Expected survival was derived using age-, sex-, and race-specific life tables from the United States ${ }^{12}$, using the Ederer II method ${ }^{13}$. Age standardized survival was calculated using International Cancer Survival Standard (ICSS) weights ${ }^{14}$ for 5 age groups.
In addition, when possible, age-specific survival was calculated for two age groups: 15-64 and 65+ years of age.

Survival estimates for which the standard error was greater than 5 percent units due to sparseness of data are not reported. Standard errors of greater than 5\% units limited the time period for which results could be reported for patients with CLL, CML, HL, and DLBCL. In addition, analysis of older patients was not conducted for ALL and CML due to the very small case numbers leading to high standard errors. Past analyses of survival for patients with HL have shown extreme variation in survival between young people with $\mathrm{HL}$ and older patients ${ }^{15}$. Possibly because of this variation, standard errors for HL overall were too high to calculate survival estimates after 10 years for all patients and for any time interval for older adults, although standard errors were actually lower for younger patients, possibly due to less heterogeneity, allowing for calculation of up to 20-year survival in this patient population.

All analyses were run on SAS Software (version 9.4, SAS, Carey, NC, USA) using macros for boomerang analyses as previously described ${ }^{10}$.

\section{Results}

Case numbers after the exclusion of DCO cases ranged from 952 cases of ALL in 2002-06 to 30,333 cases of NHL in 2012-16 (Table 1). The overall DCO rate was $0.7-1.2 \%$ for leukemias, $1.6 \%$ for $\mathrm{MM}$, and $0.2-0.8 \%$ for lymphomas, with no individual histologies with a DCO rate over $2 \%$. Case numbers increased in the later period as compared to the earlier for all malignancies. MM, CLL, and NHL were the most common malignancies in each time period, CML and ALL the least common. More men than women were diagnosed with hematologic malignancies in the relevant time periods for all malignancies examined. There was little change in gender distribution over time. As expected, median age at diagnosis was lowest for ALL and HL, highest for myeloma and CLL. The median age at diagnosis changed by one year or less between the two time periods for all conditions except for patients with CLL, for whom the median age decreased by 2 years, and for patients with ALL, for whom the median age at diagnosis increased by 4 years.

Ten and twenty-year age-standardized RS for patients with MM increased from $18.1 \%$ and $8.0 \%$, respectively, in 2002-2006 to $34.9 \%$ and $19.3 \%$, respectively, in $2012-16$ (Fig. 1a, Table 2). Survival was greater at both time points for patients age 15-64, with an increase of $+20.5 \%$ units and $+10.8 \%$ units at 10 and 20 years, respectively (Fig. $1 \mathrm{~b}$, Table 2). Both survival and changes in survival between the two-time points were lower for older patients, but an increase in RS was observed at the 10-year time point.

Relative survival was low for patients with ALL, with 10and 20 -year estimates of $13.0 \%$ and $5.6 \%$, respectively, for 
Table 1 Case numbers by histology and calendar period after removal of cases diagnosed by death certificate only (DCO) and proportion of DCO cases for 2002-2016.

\begin{tabular}{|c|c|c|c|c|}
\hline Histology & Category & $2002-2006$ & 2012-2016 & $\% \mathrm{DCO}$ \\
\hline \multirow[t]{5}{*}{ Myeloma } & All & 7508 & 10,782 & $1.6 \%$ \\
\hline & Gender & & & \\
\hline & M & 4049 (54\%) & 6076 (56\%) & \\
\hline & $\mathrm{F}$ & 3459 (46\%) & 4706 (44\%) & \\
\hline & Median age & 70 & 69 & \\
\hline \multirow[t]{5}{*}{ ALL } & All & 952 & 1384 & $0.7 \%$ \\
\hline & Gender & & & \\
\hline & M & 555 (58\%) & 805 (58\%) & \\
\hline & F & 397 (42\%) & $579(42 \%)$ & \\
\hline & Median Age & 45 & 49 & \\
\hline \multirow[t]{5}{*}{ AML } & All & 4611 & 6901 & $1.1 \%$ \\
\hline & Gender & & & \\
\hline & M & $2441(53 \%)$ & 3781 (55\%) & \\
\hline & $F$ & 2170 (47\%) & 3120 (45\%) & \\
\hline & Median Age & 68 & 69 & \\
\hline \multirow[t]{5}{*}{ CLL } & All & 7278 & 10,137 & $1.0 \%$ \\
\hline & Gender & & & \\
\hline & M & $4334(60 \%)$ & 6212 (61\%) & \\
\hline & $F$ & $2944(40 \%)$ & 3925 (39\%) & \\
\hline & Median Age & 71 & 69 & \\
\hline \multirow[t]{5}{*}{ CML } & All & 1621 & 2220 & $1.2 \%$ \\
\hline & Gender & & & \\
\hline & M & 931 (57\%) & $1254(56 \%)$ & \\
\hline & $F$ & 690 (43\%) & 966 (44\%) & \\
\hline & Median Age & 61 & 60 & \\
\hline \multirow[t]{5}{*}{$\mathrm{HL}$} & All & 3877 & 3918 & $0.4 \%$ \\
\hline & Gender & & & \\
\hline & M & 2073 (53\%) & $2213(56 \%)$ & \\
\hline & $\mathrm{F}$ & 1804 (47\%) & 1705 (44\%) & \\
\hline & Median Age & 39 & 40 & \\
\hline \multirow[t]{5}{*}{$\mathrm{NHL}$} & All & 27,555 & 30,333 & $0.8 \%$ \\
\hline & Gender & & & \\
\hline & M & $14,785(54 \%)$ & $16,762(55 \%)$ & \\
\hline & $F$ & $12,770(46 \%)$ & $13,571(45 \%)$ & \\
\hline & Median Age & 67 & 67 & \\
\hline \multirow[t]{5}{*}{ DLBCL } & All & 11,927 & 15,079 & $0.2 \%$ \\
\hline & Gender & & & \\
\hline & M & $6206(52 \%)$ & 8122 (54\%) & \\
\hline & $F$ & $5721(48 \%)$ & 6957 (46\%) & \\
\hline & Median Age & 68 & 68 & \\
\hline
\end{tabular}

$A L L$ acute lymphoblastic leukemia, $A M L$ acute myeloblastic leukemia, $C L L$ chronic lymphoid leukemia, CM chronic myeloid leukemia, HL Hodgkin lymphoma, NHL non-Hodgkin lymphoma, $D L B C L$ diffuse large B-cell lymphoma.

2002-2006 and $29.0 \%$ and $16.5 \%$ for $2012-2016$ (Fig. 2a, Table 2). However, RS was better and changes in RS between the two time points greater for patients age 15-64 (Fig. 2b, Table 2). Survival for younger patients reached a near plateau after 5 years, with only a small decrease in relative survival between 5 and 10 years and between 10 and 20 years in 2012-2016.

Ten year RS for patients with AML increased from $14.0 \%$ in $2002-2006$ to $19.0 \%$ in $2012-16$ (Fig. 2c, Table 2).

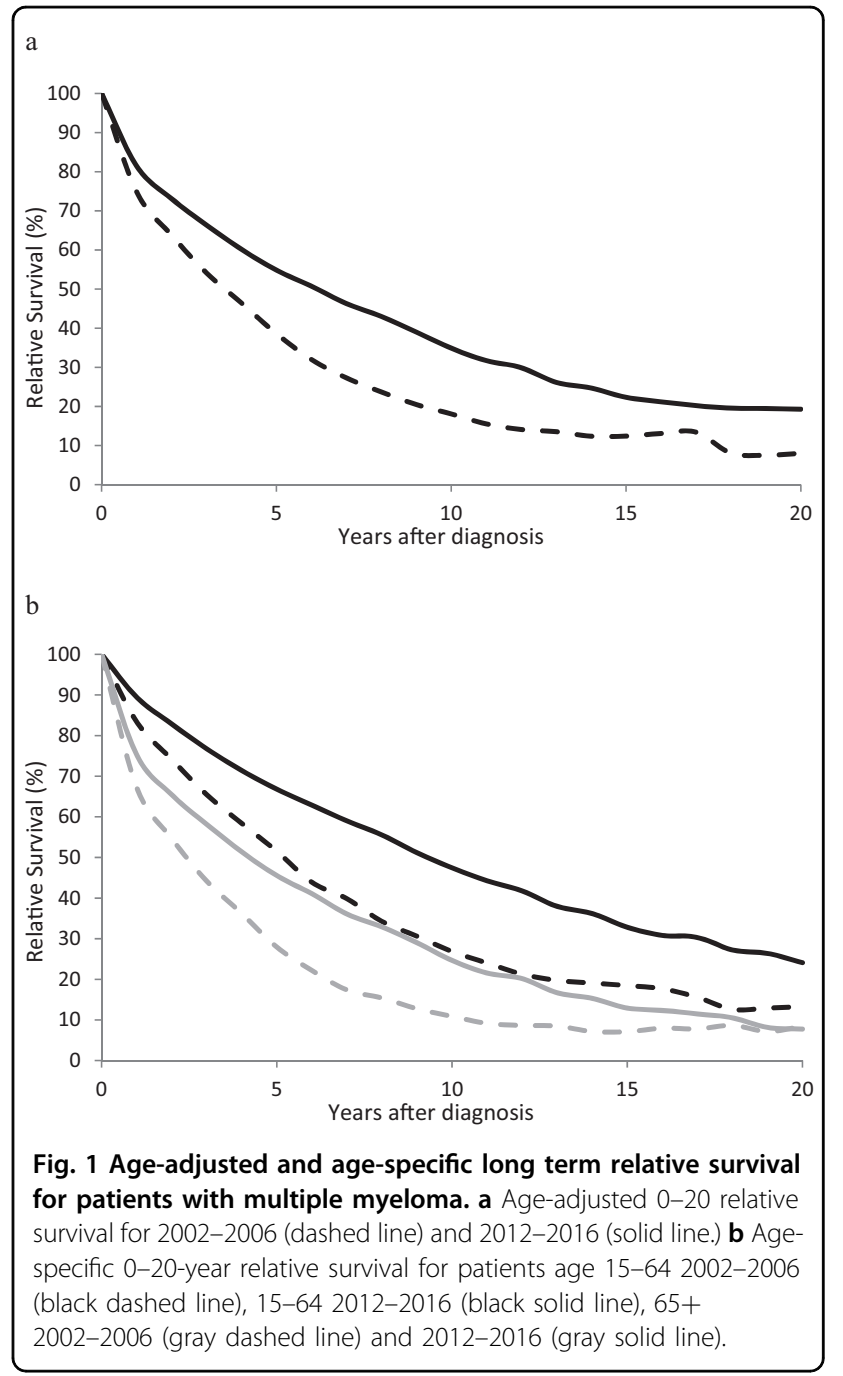

A small but persistent decrease in survival was observed between 10 and 20 years for both time periods. Survival varied considerably by age, with 10 -year survival estimates for patients age $15-64$ of $31.1 \%$ in $2002-2006$ and $39.6 \%$ in 2012-2016 as compared to 10-year estimates of 6.2\% for 2002-2006 and 6.1\% in 2012-2016 for age 65+ (Fig. 2d, Table 2). A decrease in RS was observed between 10 and 20 years for younger patients.

For patients with CLL, a major increase in RS was observed, with 10 -year survival increasing by $+9.3 \%$ units and 15-year survival increasing by $18.6 \%$ units (Fig. 3a, Table 2). Survival estimates were higher for patients age 15-64 but increases in survival were observed for both younger and older patients. (Fig. 3b, Table 2), with 15-year relative survival going from $60.3 \%$ in $2002-2006$ to $77.8 \%$ in $2012-2016$ ( $+17.5 \%$ units) for patients age 15-64 and from $45.7 \%$ to $65.7 \%$ (+20\% units) for patients age $65+$.

Changes in survival were observed for CML, with 10year estimates of RS going from $39.0 \%$ in 2002-2006 to 
Table 2 Ten and 20 year relative survival for patients with hematologic malignancies by malignancy, age, and period.

\begin{tabular}{|c|c|c|c|c|c|}
\hline Histology & Population & $2002-06$ & & 2012-16 & \\
\hline & & 10-year & 20-year & 10-year & 20-year \\
\hline & & RS (SE) & RS (SE) & RS (SE) & RS (SE) \\
\hline \multirow[t]{3}{*}{ Myeloma } & All & $18.1(1.3)$ & $8.0(1.8)$ & 34.9 (1.4) & $19.3(4.4)$ \\
\hline & $15-64$ & 26.9 (2.3) & 13.3 (2.9) & $47.4(2.1)$ & $24.1(3.1)$ \\
\hline & $65+$ & $10.9(1.4)$ & $8.4(3.8)$ & 24.7 (1.9) & $7.8(3.2)$ \\
\hline \multirow[t]{2}{*}{ ALL } & All & $13.0(2.2)$ & $5.6(1.3)$ & 29.0 (3.9) & $16.5(2.6)$ \\
\hline & $15-64$ & 36.8 (3.2) & 34.9 (3.8) & 47.0 (3.2) & $43.8(3.9)$ \\
\hline \multirow[t]{3}{*}{ AML } & All & $16.1(1.2)$ & $10.1(1.5)$ & $19.0(1.0)$ & $14.5(1.7)$ \\
\hline & $15-64$ & 31.1 (1.9) & 29.8 (2.4) & 39.6 (1.6) & $33.9(2.5)$ \\
\hline & $65+$ & $6.2(1.3)$ & 0 & $6.1(1.1)$ & $4.9(2.5)$ \\
\hline \multirow[t]{3}{*}{ CLL } & All & $67.8(2.3)$ & 37.3 (3.1) & 77.1 (1.8) & $55.9(4.2)$ \\
\hline & $15-64$ & 73.8 (2.7) & $51.2(4.4)$ & 85.8 (1.8) & $73.8(3.8)$ \\
\hline & $65+$ & 63.8 (3.2) & NA & $70.8(2.6)$ & NA \\
\hline \multirow[t]{2}{*}{ CML } & All & 39.0 (3.7) & NA & 62.1 (3.6) & NA \\
\hline & $15-64$ & $69.2(3.9)$ & NA & 84.7 (2.4) & NA \\
\hline \multirow[t]{2}{*}{$\mathrm{HL}$} & All & 50.6 (3.5) & NA & $63.9(4.0)$ & NA \\
\hline & $15-64$ & 84.9 (1.3) & 77.8 (1.9) & 88.7 (1.2) & $82.6(1.8)$ \\
\hline \multirow[t]{3}{*}{$\mathrm{NHL}$} & All & $56.5(1.0)$ & 41.5 (3.5) & $64.5(0.9)$ & $52.2(2.7)$ \\
\hline & $15-64$ & $68.4(0.9)$ & 54.6 (1.6) & $75.6(0.8)$ & $69.4(1.3)$ \\
\hline & $65+$ & $48.6(1.5)$ & 35.0 (3.9) & $56.8(1.4)$ & $42.0(3.4)$ \\
\hline \multirow[t]{3}{*}{ DLBCL } & All & 56.9 (1.8) & NA & $63.0(1.3)$ & NA \\
\hline & $15-64$ & $68.6(1.5)$ & $57.2(2.8)$ & $72.1(1.2)$ & $64.8(2.0)$ \\
\hline & $65+$ & $48.6(2.7)$ & NA & $56.3(2.0)$ & NA \\
\hline
\end{tabular}

62.1\% (+23.1\% units) in 2012-2016 (Fig. 3c, Table 2). For patients age 15-64, 10 year relative survival in 2002-2006 was $69.2 \%$ and increased to $84.7 \%$ in $2012-2016$ (Fig. 3d, Table 2).

One to 10-year and a subgroup analysis of patients age 15-64 were calculated for HL. The overall 10-year analysis showed survival of $50.6 \%$ at 10 years in 2002-06 and $63.9 \%$ at 10 years in $2012-2016$ (Fig. 4a, Table 2). Subgroup analysis of patients age 15-64 showed 10- and 20year RS estimates of $84.9 \%$ and $77.8 \%$ for $2002-2006$ and $88.7 \%$ and $82.6 \%$ for $2012-2016$ (Fig. 4b, Table 2).

Survival for patients with NHL also demonstrated a large increase between the two times examined (Fig. 4c, Table 2). Survival estimates for the later time period were greater for both older and younger patients on agespecific analysis (Fig. 4d, Table 2).

Because there are multiple subtypes of NHL with varying prognosis, survival for diffuse large B-cell lymphoma (DLBCL), the most common subtype, was calculated as well (Fig. 4e, Table 2). An increase in survival for patients with DLBCL of $+7.8 \%$ units and $+10.3 \%$ units at 10 and 15 years, respectively, was observed. RS at 10 and 15 years in 2012-2016 were 63.0 and 57.6\%. Survival increased for both younger and older patients between the two time periods, with a greater change for older patients (Fig. 4f, Table 2). For both patient groups, a rapid drop in survival the first several years after diagnosis was followed by a slow but persistent decrease in RS up to 15 years after diagnosis.

\section{Discussion}

This first comprehensive application of the newly introduced boomerang method for up-to-date long-term survival estimates showed that overall 10 - and 20-year survival increased substantially for each of the relatively common hematologic malignancies examined. Particularly impressive increases were observed for patients with CLL, CML, and NHL. However, 20-year relative survival remains under $50 \%$ for most of the malignancies examined, with the notable exception of $\mathrm{HL}$ in younger patients and plateaus in survival were observed only in a minority of cases and were not observed for NHL, in particular for DLBCL, which is generally considered curable with treatment.

A remarkable number of new treatment options have become available for the treatment of hematologic malignancies in the early 21 st century, with particularly strong progress being made for $\mathrm{MM}^{16}, \mathrm{CLL}^{17}, \mathrm{CML}^{18}$, and $\mathrm{NHL}^{19}$, though the past decade has also seen the advent of new therapeutic options for $\mathrm{ALL}^{20}, \mathrm{HL}^{21}$, and some forms of $\mathrm{AML}^{22}$. Five year RS is now greater than $50 \%$ for all of these malignancies except for the acute leukemias ${ }^{1-7,15,23,24}$ and even for these conditions, 5-year survival greater than $50 \%$ for younger patients is not unexpected $^{25,26}$. Five year RS expectations for younger patients with CML and HL as well as all patients with CLL are near to or greater than $90 \%^{2-4,15,26}$. Thus, examination of longer outcomes and the late effects of treatment are becoming more important as more patients survive the initial phase of their disease.

One notable finding is that for all conditions examined except for AML and arguably ALL in younger patients, there was a major decrease in survival between 10 and 20 years after diagnosis. This is seen even in NHL which is considered curable and thus little or no further excess mortality would be expected after the initial loss of life in the first few years after diagnosis and CML, which is generally thought to be controllable with relatively little loss of life expectancy if initial control of the disease can be established ${ }^{27}$. This decrease may be partly related to an underestimation of survival due to the advent of new therapeutic options that were not available during the 
a

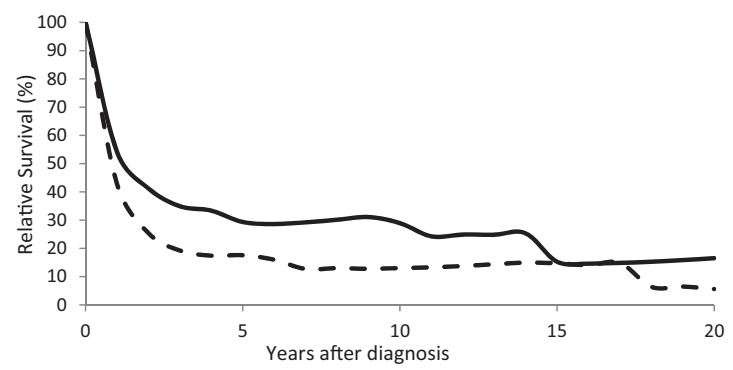

b

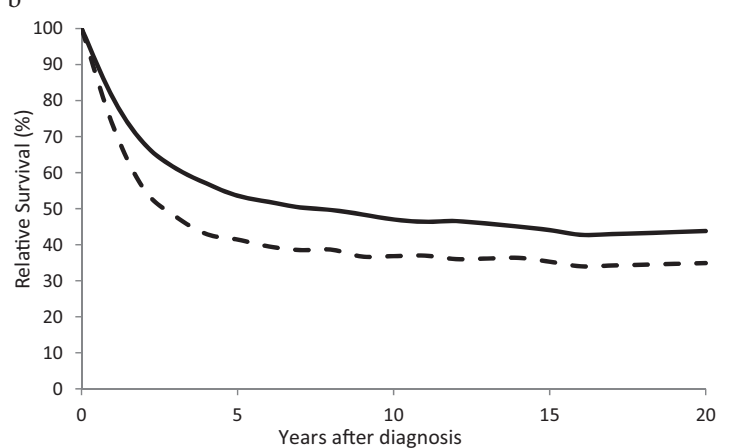

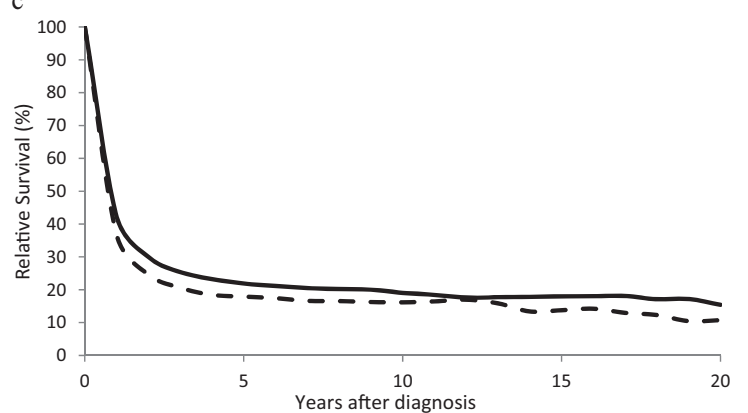

d

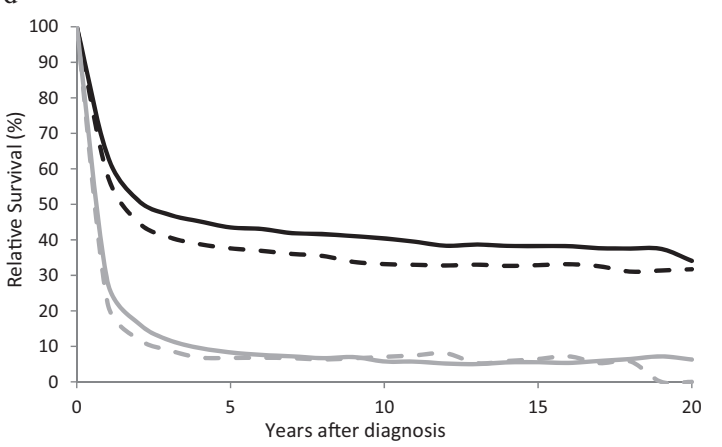

Fig. 2 Age-adjusted and age-specific long term relative survival for patients with acute leukemia. a Age-adjusted relative survival for patients with ALL in 2002-2006 (dashed line) and 2012-2016 (solid line). b Relative survival for patients age 15-64 with ALL in 2002-2006 (dashed line) and 2012-2016 (solid line). c Age-adjusted 0-20-year survival for patients with AML in 2002-2006 (dashed line) and $2012-2016$ (solid line). d Age-specific 0-20-year relative survival for patients with AML age 15-64 2002-2006 (black dashed line) and 2012-2016 (black solid line) and for patients age 65+ in 2002-2006 (gray dashed line) and 2012-2016 (gray solid line).

earlier time periods necessarily included in the survival calculation even with the boomerang method. However, a recent publication found a risk of late relapse for patients with DLBCL, suggesting that some of the decrease in survival observed may be related to late relapse ${ }^{28}$. Taken together, these results suggest that a component of late relapse or increased risk of mortality from other causes, including late effects of treatment, may contribute to the decreased long term survival even in cancers that may be curable. Given the good short term prognosis of many hematologic malignancies, consideration of long term toxicities should be a part of treatment planning for patients starting in the first line.

Prior studies in HL have demonstrated that survivors of HL have an increased risk of cardiac disease and other malignancies ${ }^{29}$. In addition, an increased risk of secondary malignancy has been observed in patients with MM, especially after treatment with immunomodulatory agents $^{30}$. Thus, it is likely that some of the decrease in survival between 10 and 20 years observed with most malignancies is related to increased risk of other causes of mortality. Increased monitoring and aggressive screening of survivors of hematologic malignancies for conditions where screening has been demonstrated to reduce mortality or morbidity and is appropriate for the specific patient may help decrease this excess mortality. In addition, while continued efforts to find curative treatment for these malignancies is important, an increased focus on potential long term sequelae of treatment, i.e. the potential for increased risk of cancer due to DNA damaging chemotherapy or of heart disease due to radiation to the chest, must be considered when evaluating potential treatment regimens.

One unexpected finding was that relative survival estimates did not always decrease consistently with time. For example, patients with ALL had an apparent increase in relative survival at about 9 and 14 years follow-up in the 2012-16 time period. This apparent increase in relative survival is likely due to small case numbers and statistical "noise". It is possible, but less likely, that better than average medical monitoring or better health habits in survivors of cancer led to a decreased risk of death due to other causes at some time points.

Strengths of our study include the use of the large and long running SEER database, which allows for examination of long term survival in patients with hematologic malignancies, most of which are relatively rare. In addition, the use of the boomerang method allows for 


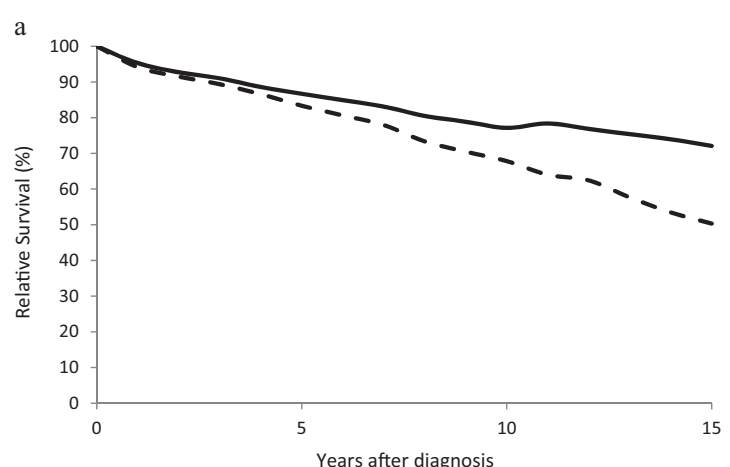

$\mathrm{b}$

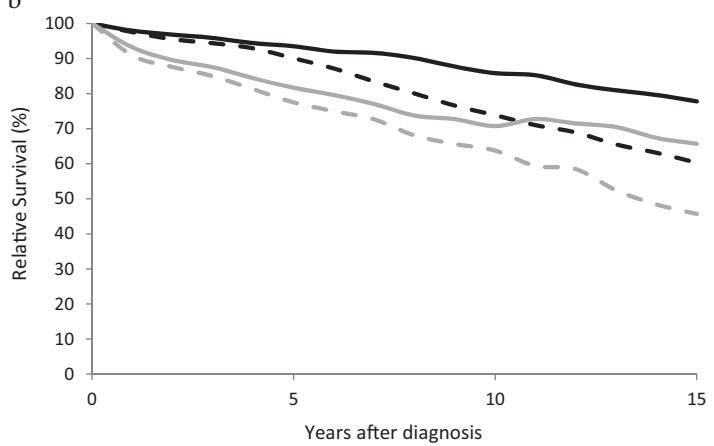

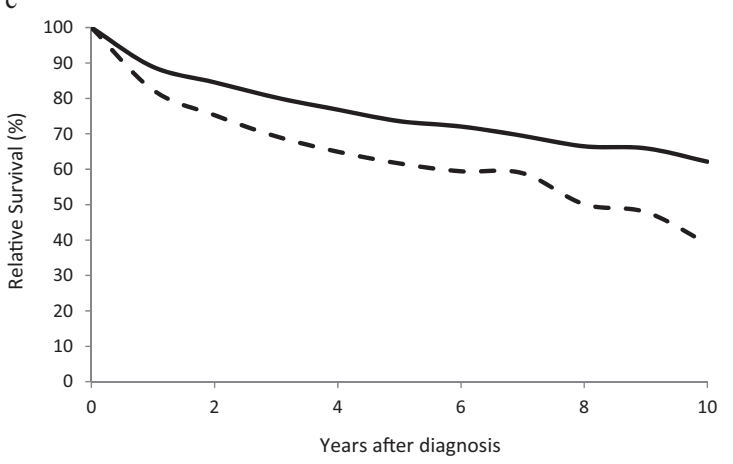

d

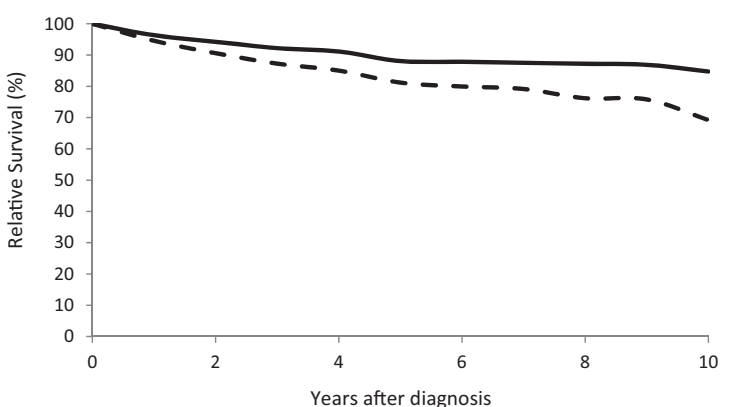

Fig. 3 Age-adjusted and age-specific long term relative survival for patients with chronic leukemia. a Age-adjusted 0-20 relative survival for patients with CLL in 2002-2006 (dashed line) and 2012-2016 (solid line). b Age-specific 0-20-year relative survival for patients with CLL age 15-64 in 2002-2006 (black dashed line) and 2012-2016 (black solid line) and for patients age 65+ in 2002-2006 (gray dashed line) and $2012-2016$ (gray solid line). c Age-adjusted 0-20-year survival for patients with CML in 2002-2006 (dashed line) and 2012-2016 (solid line). d Age-specific 0-10-year relative survival for patients with CML age 15-64 in 2002-2006 (black dashed line) and 2012-2016 (black solid line) and for patients age 65+ in 2002-2006 (gray dashed line) and 2012-2016 (gray solid line).

estimates of survival that are expected to be much closer to later observed survival experience of recently diagnosed patients compared to the use of older methods such as cohort analysis, in a condition where survival is changing rapidly.

In considering our results, some limitations should be kept in mind. First, the SEER database does not include information on chemotherapy use and whether treatment was provided with curative or palliative intent, so any correlation between changes in therapy and survival is necessarily indirect. Second, the SEER database does not include other information of potential prognostic significance such as white blood cell count at diagnosis for acute leukemia or prognostic score for NHL. Therefore, it is not possible to state definitively that there have been no changes in these factors at diagnosis which might influence outcomes. Third, even with the use of the boomerang method, it is likely that long term survival estimates are lower than the actual survival of patients diagnosed in the current era due to the necessity of including patients diagnosed before the advent of many modern therapeutic agents. Fourth, even in the 3 years between the most recent data in SEER and the present, a number of new therapeutic options have become available and thus the full effect of changes in therapy on the population level is not yet evident. Finally, in this paper, we did not consider subgroup analyses of potential importance (i.e., race, gender, and cause of death). In addition, the reasons for late mortality may be changing as treatment shifts from use of cytotoxic chemotherapy only to use of combination cytotoxic therapy, immune therapy, and small molecule enzyme inhibitors.

In summary, 10- and 20-year RS has substantially increased for common hematologic malignancies between 2002-2006 and 2012-2016. Long-term survival is still low and no plateau in survival was observed for most conditions, suggesting that there may be continued late mortality, either from late effects of the disease or therapy. Further research to determine the etiology of these issues and any changes over time in the causes of late mortality as well as to minimize therapeutic toxicity is needed to ensure optimal outcomes. 

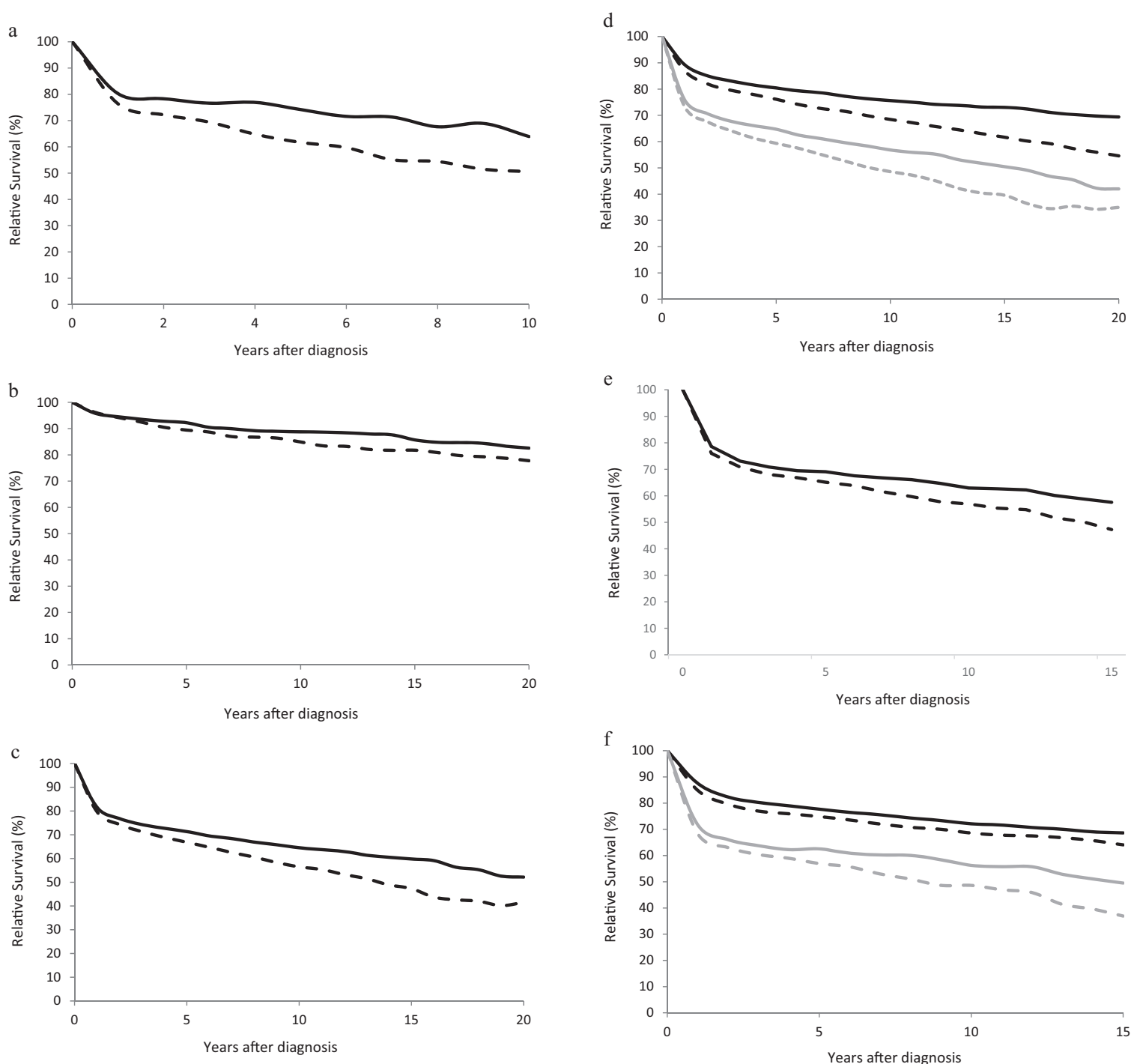

Fig. 4 Age-adjusted and age-specific long term relative survival for patients with HL and NHL. a Relative survival for patients with HL in 2001-2005 (dashed line) and 2012-2016 (solid line). b Relative survival for patients age 15-64 with HL in 2002-2006 (dashed line) and 2012-2016 (solid line). c Age-adjusted 0-20 year survival for patients with NHL in 2002-2006 (dashed line) and 2012-2016 (solid line). d Age-specific 0-20-year relative survival for patients with NHL age 15-64 in 2002-2006 (black dashed line) and 2012-2016 (black solid line) and for patients age 65+ in 2002-2006 (gray dashed line) and 2012-2016 (gray solid line). e Age-adjusted 0-20-year survival for patients with DLBCL in 2002-2006 (dashed line) and 2012-2016 (solid line). f Age-specific 0-20-year relative survival for patients with DLBCL age 15-64 in 2002-2006 (black dashed line) and 2012-2016 (black solid line) and for patients age 65+ in 2002-2006 (gray dashed line) and 2012-2016 (gray solid line).

\section{Acknowledgements}

Ethics approvals: Data are derived from de-identified database. In addition, ethics approval for the Krebshilfe-Projeket, including international comparisons, was granted by the DKFZ ethics committee. This work was supported in part by a grant from the German Cancer Aid (Deutsche Krebshilfe, no. 108257) and a visiting scientist grant from the German Cancer Research Center to D.P.

\section{Author details}

${ }^{1}$ Division of Clinical Epidemiology and Aging Research, German Cancer Research Center (DKFZ), Heidelberg, Germany. ${ }^{2}$ Division of Preventive Oncology, German Cancer Research Center (DKFZ) and National Center for Tumor Diseases (NCT), Heidelberg, Germany. ${ }^{3}$ German Cancer Consortium (DKTK), German Cancer Research Center (DKFZ), Heidelberg, Germany

\section{Author contributions}

All authors contributed to the design of the analysis, interpretation of the data, and read and approved the final paper. In addition, D.P. and L.J. performed the analysis. DP wrote the paper. L.J. and H.B. provided critical feedback on the paper

\section{Conflict of interest}

The authors declare that they have no conflict of interest.

\section{Publisher's note}

Springer Nature remains neutral with regard to jurisdictional claims in published maps and institutional affiliations. 
Supplementary Information accompanies this paper at (https://doi.org/ 10.1038/s41408-020-0323-4).

Received: 2 August 2019 Revised: 29 November 2019 Accepted: 3 December 2019

Published online: 13 May 2020

\section{References}

1. Pulte, D., Jansen, L., Castro, F. A. \& Brenner, H. Changes in the survival of older patients with hematologic malignancies in the early $21^{\text {st }}$ century. Cancer $\mathbf{1 2 2}$ 2031-40. (2016).

2. Gunnarsson, N. et al. Population-based assessment of chronic myeloid leukemia in Sweden: striking increase in survival and prevalence. Eur. J. Haematol. 97, 387-92. (2016).

3. Pulte, D. et al. Population level survival of patients with chronic myelocytic leukemia in Germany compared to the US in the early $21^{\text {st }}$ century. J. Hematol Oncol. 6, 70 (2013).

4. Pulte, D., Redaniel, M. T., Bird, J. \& Jeffreys, M. Survival for patients with chronic leukemias in the US and Britain: age-related disparities and changes in the early 21st century. Eur. J. Haematol. 94, 540-545 (2015).

5. Pozzi, S. et al. Survival of multiple myeloma patients in the era of novel therapies confirms the improvement in patients younger than 75 years: a population-based analysis. Br. J. Haematol. 163, 40-46 (2013).

6. Pulte, D. et al. Trends in survival of multiple myeloma patients in Germany and the United States in the first decade of the $21^{\text {st }}$ century. Br. J. Haematol. 171, 189-96. (2015).

7. Pulte, D., Gondos, A. \& Brenner, H. Ongoing improvement in outcomes for patients diagnosed as having non-Hodgkin lymphoma from the 1990s to the early $21^{\text {st }}$ century. Arch. Intern. Med. 168, 469-476 (2008).

8. Johnson, J. R. et al. Approval summary: Imatinib mesylate capsules for treatment of adult patients with newly diagnosed Philadelphia chromosomepositive chronic myeologenous leukemia in chronic phase. Clin. Cancer Res. 9 , 1972-1979 (2003).

9. Brenner, H. \& Hakulinen, T. Very-long-term survival rates of patients with cancer. J. Clin. Oncol. 20, 4405-4409 (2002).

10. Brenner, H. \& Jansen, L. Timely disclosure of progress in long-term cancer survival: the boomerang method substantially improved estimates in a comparative study. J. Clin. Epidemiol. 70, 224-232 (2016).

11. Surveillance, Epidemiology, and End Results (SEER) Program (www.seer.cancer. gov) Research Data (1973-2012), National Cancer Institute, DCCPS, Surveillance Research Program, Cancer Statistics Branch, released April 2016, based on the November 2015 submission.

12. Arias, E. United States life tables, 2011. Nat/ Vital. Stat. Rep. 64, 1-63 (2015).

13. Ederer $F ., H$. H. Instructions to IBM 650 programmers in processing survival computations. (National Cancer Institute, Bethesda, MD, 1959).
14. Corazziari, I., Quinn, M. \& Capocaccia, R. Standard cancer patient population for age standardizing survival ratios. Eur. J. Cancer 40, 2307-2316 (2004).

15. Brenner, H., Gondos, A. \& Pulte, D. Ongoing improvement in long-term survival of patients with Hodgkin disease at all ages and recent catch-up of older patients. Blood 111, 2977-2983 (2008).

16. Moreau, P., Attal, M. \& Facon, T. Frontline therapy of multiple myeloma. Blood 125, 3076-3084 (2015).

17. Nabhan, C. \& Rosen, S. T. Chronic lymphocytic leukemia: a clinical review. JAMA 312, 2265-2276 (2014).

18. Baccarani, M., Castagnetti, F., Gugliotta, G. \& Rosti, G. A review of the European LeukemiaNet recommendations for the management of CML. Ann. Hematol. 94(Suppl2), S141-S147 (2015).

19. Sawas, A., Diefenbach, C. \& O'Connor, O. A. New therapeutic targets and drugs in non-Hodgkin's lymphoma. Curr. Opin. Hematol. 18, 280-287 (2011).

20. Jabbour, E., O'Brien, S., Konopleva, M. \& Kantarjian, H. New insights into the pathophysiology and therapy of adult acute lymphoblastic leukemia. Cancer 121, 2517-28. (2015).

21. Eichenauer, D. A. \& Engert, A. Antibodies and antibody-drug conjugates in the treatment of Hodgkin lymphoma. Eur. J. Haematol. 93, 1-8 (2014).

22. Cull, E. H. \& Altman, J. K. Contemporary treatment of APL. Curr. Hematol. Malig. Rep. 9, 193-201 (2014).

23. Pulte, D. et al. Survival of adults with acute lymphoblastic leukemia in Germany and the United States. PLoS ONE. 9, e85554 (2014).

24. Pulte, D. et al. Survival in patients with acute myeloblastic leukemia in Germany and the United States: major differences in survival in young adults. Int. J. Cancer 139, 1289-1296 (2016).

25. Pulte, D., Redaniel, M. T., Jansen, L., Brenner, H. \& Jeffreys, M. Recent trends in survival of adult patients with acute leukemia: overall improvements, but persistent and partly increasing disparity in survival of patients from minority groups. Haematologica. 98, 222-229 (2013).

26. Pulte, D., Gondos, A. \& Brenner, H. Trends in survival after diagnosis with hematologic malignancy in adolescence or young adulthood in the United States, 1981-2005. Cancer 115, 4973-4979 (2009).

27. Bower, $\mathrm{H}$. et al. Life expectancy of patients with chronic myeloid leukemia approaches the life expectancy of the general population. J. Clin. Oncol. 34, 2851-2857 (2016)

28. Stephens, D. M. et al. Continued risk of relapse independent of treatment modality in limited-stage diffuse large B-cell lymphoma: final and long-term analysis of Southwest Oncology Group Study S8736. J. Clin. Oncol. 34, 2997-3004 (2016).

29. Yeh, J. M. \& Diller, L. Pediatric Hodgkin lymphoma: trade-offs between shortand long-term mortality risks. Blood 120, 2195-2202 (2012).

30. Areethamsirikul, N. \& Reece, D. E. The risk of secondary primary malignancies after therapy for multiple myeloma. Leuk. Lymphoma. $\mathbf{5 6}$ 3012-3021 (2015). 